\title{
THE ROLE OF COGNITIVE SCHEMATA IN THE DEVELOPMENT OF POSTTRAUMATIC STRESS DISORDER: RESULTS OF CROSS-SECTIONAL AND LONGITUDINAL STUDIES
}

\section{BOHDAN DUDEK and WIESŁAW SZYMCZAK}

\author{
University of Łódź, Łódź, Poland \\ Institute of Psychology
}

\begin{abstract}
Objective: There are two theories (by Horowitz and by Foa) which attempt at explaining the process of posttraumatic stress disorder (PTSD) development by information dissonance. The purpose of the present study was to verify these theories via cross-sectional and longitudinal studies. Materials and Methods: The study based on a cross-sectional design was performed on a representative group of Polish firefighters. The study using a longitudinal protocol was conducted among police officers. The level of PTSD was assessed using the Questionnaire for PTSD Measurement (K-PTSD). The sense of coherence (SOC) was measured with the Polish adaptation of the Orientation to Life Questionnaire by A. Antonovsky. Results: In the cross-sectional study, the correlation coefficients between K-PTSD and SOC were $-0.35(\mathrm{p}<0.001)$ in the group of firefighters and -0.47 ( $p<0.001)$ in that of police officers. In the longitudinal study, the relationship between SOC and PTSD symptoms appeared to be curvilinear and had the U-letter shape. Therefore, we used ANOVA for statistical analysis. Unfortunately, the $\mathrm{F}(2.31)=1.900$ and $\mathrm{p}>0.05$ indicated that the tendency we observed was not statistically significant. Conclusions: We would like to propose two possible explanations for the difference in the relationships between SOC and PTSD symptoms obtained in the studies employing the cross-sectional and the longitudinal protocols.
\end{abstract}

Key words:

Cross-sectional study, Longitudinal study, SOC, PTSD, Firefighters, Police officers

\section{INTRODUCTION}

In view of the high level of human exposure to traumatic events [1-3] nowadays, the scientists are more and more interested in elucidating the psychological sequels of participation in such events. These sequels may assume a form of a posttraumatic stress disorder (PTSD). It is diagnosed in an individual who participated in a traumatic event and after one month the following symptoms are still lasting:

(a) intrusive images of the event in day or night dreams:

(b) perpetual avoiding of stimulus related to trauma, or numbing: (c) level of arousal higher than before participation in the traumatic event [4]. There are several theories explaining the PTSD development. Some of them refer to a neurological mechanism. Charney and co-workers [5] claim that neurological circuits are developed in the brain that are responsible for the formulation and lasting of the traumatic event sequels. Experimental and clinical studies provide evidence for connections between such brain structures like an amygdale, a locus coeruleus, a thalamus and an

Received: July 8, 2010. Accepted: September 8, 2010.

Address reprint request to B. Dudek, Institute of Psychology, University of Łódź, ul. Smugowa 10/12, 91-433 Lódź, Poland (e-mail: bdudek@uni.lodz.pl). 
anxious reaction. Some researchers use the psychoanalytic concepts to explain PTSD development. They pay special attention to the traumatic events which happened in early childhood because anxious memories and negative emotions connected with these events may be activated during participation in a given traumatic condition [6]. The learning theory is another concept used to elucidate the mechanism of developing PTSD symptoms. Classical conditioning is postulated as an explanation that the thoughts, cognitions, words etc, which are neutral before one's participation in a traumatic event, start to arouse anxiety after the exposure to such an event. Instrumental conditioning is proposed to explain the development of avoiding reactions related to trauma [7]. Some cognitive psychologists are inclined to apply the cognitive schemata theory to the explanation of PTSD development. These researchers assume that information coming from a traumatic event does not fit the information contained in the core cognitive schemata which were fixed in the individual's mind during his/ her lifespan. The cognitive schemata which pertain to the external world and oneself - that the world is safe, fair and controllable, that people are trustful, and that he/she has enough competencies to cope with different difficulties and to manage his/her own emotional problems - belong to the core schemata. The individual who is exposed to a traumatic event has difficulties with assimilating and accommodating the information coming from this event to his/her own previously acquired and well-fixed cognitive schemata. One takes effort to do it but cannot succeed. At the end of this process, some behaviours classified as the PTSD symptoms are manifested, which indicates that the new information related to the traumatic event has not been assimilated into the existing cognitive schemata.

There are two theories which attempt at explaining the process of PTSD development by the above-mentioned information dissonance. Horowitz, who is the author of the miss-match theory [8] assumed that the highest difficulties in transforming the trauma-related information will be experienced by the persons characterised by extremely positive cognitive schemata related to the world and themselves. Those individuals will be moved by strong emotions, which make them deny the information coming from a traumatic situation. We can expect that this category of individuals will have difficulties in internalizing the trauma-related information and assimilating it into fixed, core cognitive schemata. Therefore, possessing extremely positive beliefs about the world is a risk factor for the development of the PTSD symptoms.

Foa does not accept all the assumptions of the theory by Horowitz [7]. She points to the empirical results of many studies which indicate another group of individuals who have a high potential to develop PTSD symptoms. This group includes the persons who had had some traumatic experiences earlier in their life, e.g. during childhood and hence they could not develop positive cognitive schemata about the world and themselves. The schemata built up by those persons are not in dissonance with the information coming from a given traumatic event and, according to the Horowitz theory, they should faster regain the normal status as they do not need much time to assimilate the new information. Foa proposed a theory which is complementary to the one by Horowitz. She claims that two groups of individuals will be more inclined to develop PTSD symptoms. These are: (a) persons with extremely positive cognitive schemata about the world and themselves - this assumption derives from the Horowitz theory, and (b) persons who have negative beliefs about the external world and their own competencies to overcome the different barriers and traps that come across in their lives. It is presumed that the individuals from group (b) will develop PTSD symptoms because their cognitive schemata are related to anxiety and hence the information coming from a traumatic event can evoke an even higher anxiety. The group which is the most resistant 
to PTSD are the individuals who have flexible beliefs about the world (it can sometimes be safe and sometimes dangerous) and their competencies (one can usually cope with difficult problems and situations, but may fail at times as well). In our research practice we have not thus far come across any publications that would compare these two theories.

\section{OBJECTIVES AND HYPOTHESES}

To verify the validity of both theories we should discriminate the subjects presenting positive and negative cognitive schemata about the world and themselves. Having considered the issue in detail we have arrived at a conclusion that Antonovsky's Orientation to Life Questionnaire, measuring the sense of coherence understood as a global orientation to life, would be the best instrument [9]. The SOC questionnaire consists of three following components: (1) comprehensibility - it is a sense that everything which happens to persons in their lives is understood and can be foreseen because the world is ordered and cohesive; (2) manageability - it is a sense that a given individual has got enough competence to cope with all the problems and that he/she is very effective in controlling all the affairs one deals with; (3) meaningfulness - it is a sense that solving difficult problems is worth engaging and it is reasonable to exert a strong effort to approach the worthy things. SOC measurement makes it possible to verify the Horowitz and Foa hypotheses because the subjects with high SOC represent individuals with positive schemata about the world and themselves, and the low SOC subjects are among those with negative cognitive schemata.

We have put forward the following working hypotheses:

- $\mathrm{H}_{1}$ - there will be a positive correlation between SOC and the PTSD symptoms (confirmation of this hypothesis will indicate that the Horowitz theory is valid).
- $\mathrm{H}_{2}$ - the high and low SOC subjects will be more inclined to develop the PTSD symptoms than those with moderate SOC (confirmation of this hypothesis will indicate the validity of the Foa theory).

\section{MATERIALS AND METHODS}

The verification of the above mentioned hypotheses was based on the results obtained in two earlier studies. In the first one, the subjects were a representative group of Polish firefighters $(\mathrm{N}=974$; mean age 33.5 years and $\mathrm{SD}=5.9)$. It was a cross-sectional study ${ }^{1}$. The subjects were asked to recall all the traumatic events they had experienced over a lifetime when performing their professional duties. If they had experienced more than one such event, they were instructed to choose the most dramatic event and write down a short description of this situation. There were $839(85 \%)$ subjects who reported participating in at least one event of this kind. Some of them did develop the PTSD symptoms.

The second study was conducted according to a longitudinal design and was divided into two stages. At the first stage, which resembled a cross-sectional study, the subjects were a group of police officers $(\mathrm{N}=833$; mean age 28.5 years and $\mathrm{SD}=5.59$; male subjects only). In that group, $453(57.4 \%)$ subjects participated in one or more traumatic events on duty. The PTSD symptoms were measured only in that group of subjects. At the second stage, we tried to get into contact with all the subjects examined during the previous stage but we sometimes failed for a number of reasons including retirement, moving to a new residence, refusal, vacation, etc. Eventually, we managed to examine 399 police officers who had been the subjects in the first stage of the study (mean age 27.2 years and $\mathrm{SD}=7.34$ ). The second stage examinations were conducted 12-14 months after the first series. The subjects

\footnotetext{
1 The study was supported through the grant from the Polish Committee for Scientific Research No. PBZ010-11.
} 
were asked to report if they had experienced any traumatic event during this period. Of the total number of these subjects, $36(9 \%)$ reported that they had participated in a traumatic situation when performing their duties².

In the study on firefighters and during the first stage of the study on police officers, we used a large set of methods measuring different variables. The results of these studies are discussed elsewhere [10,11]. In the present report, we will be analysing the results obtained using two methods. The level of PTSD symptoms was measured with the Questionnaire for PTSD Measurement (K-PTSD) based on the PTSD-I by Watson et al. [12], but adapted for use in a group survey. The K-PTSD consists of 17 questions covering all the PTSD symptoms as listed in the definition presented in DSM IV [4]. The subjects were asked to assess the severity and frequency of each symptom on a 7-point rating scale. The reliability of the Polish version of the questionnaire was at an acceptable level (Cronbach alfa $=0.90)$ [13]. The sense of coherence (SOC) was assessed using a Polish version of the Orientation to Life Questionnaire by Antonovsky [9]. The SOC questionnaire consists of 27 items measuring the three following components: comprehensibility, manageability and meaningfulness. The Cronbach alfa coefficients for the particular components and the total score were $0.78,0.72,0.68$ and 0.92 , respectively [14].

\section{RESULTS}

Table 1 presents the distribution of results for the three groups of subjects: (1) representative population of Polish firefighters, (2) police officers - stage I, (3) police officers - stage II. In the first two groups, both variables were measured at the same time. Thus, the results of the examinations in these groups can be used to find a relationship between SOC and PTSD symptoms if we

\footnotetext{
2 The study was supported by the Polish Committee for Scientific Research; Grant No. SPR04.10.331.
}

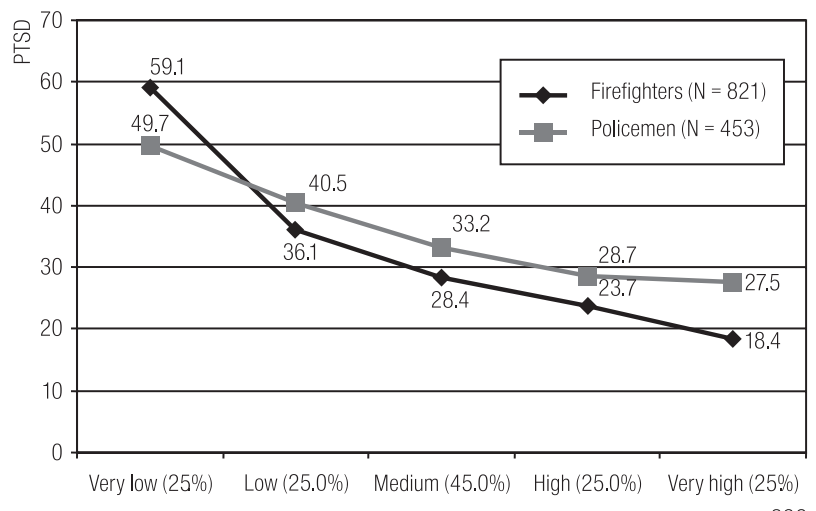

SOC — sense of coherence; PTSD — posttraumatic stress disorder.

Fig. 1. Relationship between PTSD symptoms and SOC: a cross-sectional study.

apply a cross-sectional protocol. In the third group, the level of PTSD symptoms was measured 12-14 months since SOC measurement. The findings in Table 1 indicate that there is no difference in the level of SOC between the study groups, but that there is a very large difference in the level of PTSD symptoms between the groups of police officers. This difference can be explained if one takes into account that the memory of the traumatic event was very fresh for the police officers examined during the second stage of the study.

To verify the $\mathrm{H}_{1}$ hypothesis, the firefighters and the police officers participating in stage I of the study were divided into five subgroups on the basis of the percentage distribution of SOC results. We abstracted two extreme groups comprising $2.5 \%$ of the subjects with the highest and lowest SOC score, referred to as the high SOC (25\%) and low SOC (25\%) subjects, and one subgroup, with SOC results located in the middle part of the distribution, hence termed the medium SOC subjects (45\%). The relationship between SOC and PTSD symptoms is displayed in Figure 1. We can notice an almost linear relationship, which makes it possible to calculate Pearson's r coefficient to determine the correlation power between the variables. The correlation coefficients were $-0.35(p<0.001)$ for the firefighters 
Table 1. Means (M) and standard deviations (SD) of SOC and PTSD symptoms in three groups of subjects exposed to traumatic events

\begin{tabular}{lccccc}
\hline \multirow{2}{*}{ Groups of subjects } & \multirow{2}{*}{$\mathrm{N}$} & \multicolumn{3}{c}{ SOC } & \multicolumn{2}{c}{ PTSD } \\
\cline { 3 - 6 } & & $\mathrm{M}$ & $\mathrm{SD}$ & $\mathrm{M}$ & $\mathrm{SD}$ \\
\hline Representative group of Polish firefighters & 833 & 143.9 & 20.20 & 34.4 & 14.78 \\
Police officers - stage I of the study & 453 & 142.9 & 22.17 & 29.5 & 13.40 \\
Police officers - stage II of the study & $35^{*}$ & 143.8 & 19.57 & 40.2 & 24.30 \\
\hline
\end{tabular}

SOC - sense of coherence; PTSD - posttraumatic stress disorder.

* One subject resigned from participation during the examination.

and -0.47 ( $p<0.001)$ for the police officers. We can observe a similar tendency in both the study groups, although they were examined in independent studies. This indicates a true tendency, not an artefact.

In the group of police officers who were examined during stage II of the study, we can distinguish three subgroups only because of the small number of subjects. These were the subjects with the highest SOC (27.5\%), the lowest SOC (27.5\%), and the medium SOC (45\%). In Figure 2, we can notice a curvilinear relationship between the SOC and the PTSD symptoms; it has the $\mathrm{U}$ letter shape. The finding indicates that the highest and lowest SOC subjects presented with the highest level of PTSD symptoms while the medium SOC subjects were characterized by the lowest level of PTSD. We applied the analysis of variance to evaluate whether this tendency was statistically significant or not. Unfortunately, $\mathrm{F}(2.31)=1.900, \mathrm{p}>0.05$ (Table 2) implies that the

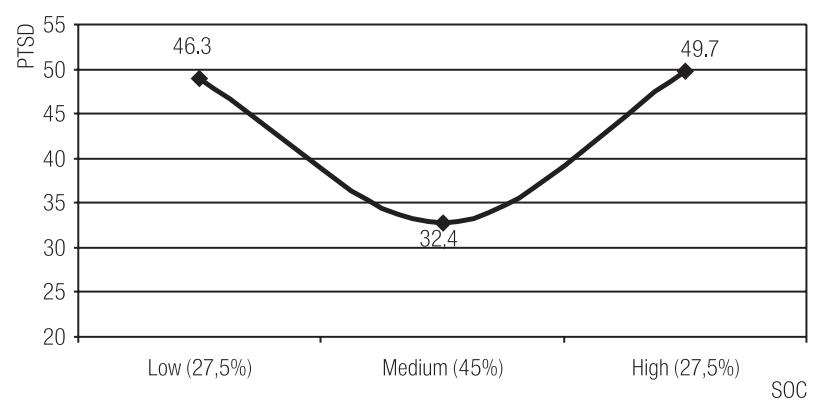

Abbreviations as in Table 1.

Fig. 2. Relationship between PTSD symptoms and SOC: a longitudinal study.
Table 2. Relationship between SOC and PTSD symptoms (ANOVA)

\begin{tabular}{lrccccc}
\hline SOC level & N & M & SD & df & F & p \\
\hline Low & 9 & 46.3 & 27.96 & 2.31 & 1.900 & 0.167 \\
Medium & 16 & 32.4 & 21.01 & & & \\
High & 9 & 49.7 & 23.20 & & & \\
\hline
\end{tabular}

Abbreviations as in Table 1.

tendency appeared not to be significant. We suppose that the factor accounting for this result may have been the small number of subjects.

\section{DISCUSSION AND CONCLUSIONS}

From the findings in Figures 1 and 2 we can see that there are different relationships between SOC and PTSD symptoms. The results obtained in the cross-sectional study point to protective role of SOC in the development of the PTSD symptoms. Neither of our working hypotheses was confirmed. In the $\mathrm{H}_{1}$, following the Horowitz theory we expected that SOC will be a risk factor, but the results of our study revealed that SOC played a completely different role. It appeared to be an individual resource. Because such a function of SOC was found in two independent studies, the results provide a strong support for the Antonovsky's concept [9]. He was right when he wrote about the protective role of SOC in the stress process regardless of the kind of stress. 
A different relationship between SOC and PTSD symptoms was found in the third study, conducted according to a longitudinal protocol. The obtained results assured us that the relationship between both the variables is a curvilinear one. The high and low SOC subjects were more inclined to develop PTSD symptoms than the medium SOC subjects. If we had more subjects participating in the study, the $\mathrm{H}_{2}$ would be confirmed at the level of statistical significance. Considering this explanation and the power of evidence obtained in the longitudinal study, we presume that the Foa theory is valid.

The main problem that we anticipated in this research seems to be resolved because based on the study results we could assess which theory on the development of PTSD symptoms is right. However, another important question needs to be answered, namely of how to explain the different relationships between SOC and PTSD symptoms obtained in the studies employing two different protocols: a cross-sectional and longitudinal one. We would like to propose two possible explanations to this finding: the first one refers to a methodological factor, and the other one to a mechanism of the SOC impact on PTSD development. The tendency noted in the cross-sectional study is due to a common variance of both variables. It is a factor responsible for the correlation between two variables, especially if the researchers use self-report methods to measure these variables. It is well known that people are inclined to avoid cognitive dissonance and this is the reason why the subjects respond to questionnaire items in a very cohesive way. The sense of coherence consists of well-fixed beliefs on the world, other individuals and one's own competencies, and all these beliefs are an important part of somebody's personality. To avoid cognitive dissonance, the high SOC subjects, who are convinced that the world is safe and everything is under control, are inclined to answer to questions about a traumatic event in a specific way: the event is not very dangerous, and the consequences of taking part in the event are not very painful. The low SOC subjects have opposite beliefs and are inclined to answer the questions in an opposite way.

As the other explanation we offer a specific impact of SOC on the process of developing the PTSD symptoms. The signals coming from a traumatic event are so terrifying that SOC can have no impact on receiving this piece of information. SOC can influence processing of information on the traumatic situation and coping with mental sequels of participation in the traumatic event. It is well documented that the high SOC people tend to more frequently look for social support through telling other people about the trauma, and they seem to use more effective coping strategies. The characteristics of the high SOC people includes a faster recovery from trauma, but to be effective, the influence of SOC must be long lasting. In the cross-sectional study, the interval between exposure to a traumatic event and the examination was longer than in the longitudinal one. This may be the second reason why we noted a protective role of SOC in the former study. However, neither of the explanations we propose accounts for the direction of the relationship we found in the latter study. To explain this, we would rather have to apply the Foa theory.

\section{REFERENCES}

1. Breslau N, Davis GC, Andreski P. Risk factors for PTSD-related traumatic events: a prospective analysis. Am J Psychiatry 1995;152(4):529-35.

2. Kessler BC, Mc Gonagle KA, Zhao S. Lifetime and 12-month prevalence of DSM-III-R psychiatric disorders in the United States: results from the National Comorbidity Survey. Arch Gen Psychiatry 1994;51(8):8-19.

3. Creamer M, Burgess P, McFarlane AC. Post-traumatic stress disorder: findings from the Australian National Survey of Mental Health and Well-being. Psychol Med 2001;34:1237-47.

4. American Psychiatric Association. Diagnostic and Statistical Manual of Mental Disorders. Washington, DC: Author; 1994. 
5. Charney DS, Deutch AY, Southwich SM, Krystal JH. Neural circuits and mechanism of post-traumatic stress disorder. In: Friedman MJ, Charney DS, Deutch AY, editors. Neurobiological and clinical consequences of stress. from normal adaptation to post-traumatic stress disorder. Philadelphia: Lippincott-Raven Publisher; 1995. p. 271-90.

6. Van der Kolk BA. The compulsion to repeat trauma. Psychiatr Clin North Am 1989;12(2):389-411.

7. Foa EB, Rothbaum BO. Treating the trauma of rape. Cognitive-behavioral therapy for PTSD. New York: Guilford Press; 1989.

8. Horowitz MJ. Stress Response Syndromes. Northvale, New Jersey, London: Jason; 1986.

9. Antonovsky A. Unraveling the mystery of health. How people manage stress and stay well. Warszawa: Fundacja IPiN; 1995.

10. Dudek B, Koniarek J. Personality determinants of the development of posttraumatic stress disorder. In: Strelau J, editor. Personality and extreme stress. Gdańsk: GWP; 2004. p. 183-98 [in Polish].

11. Dudek B. Post-traumatic Stress Disorder. Gdańsk: GWP; 2003 [in Polish].

12. Watson ChG, Juba MP, Manifold V, Kucala T, Anderson PE. The PTSD Interview: Rationale, Description, Reliability, and Concurrent Validity of a DSM III Based Technique. J Clin Psychol 1991;47(2):179-88.

13. Koniarek J, Dudek B, Szymczak M. Questionnaire measuring post-traumatic stress disorders (K-PTSD) - application of the PTSD-Interview by Ch. Watson et al. in a group survey. Przegl Psychol 2000;43(2):205-16 [in Polish].

14. Koniarek J, Dudek B, Makowska Z. The Sense of Coherence Questionnaire (SOC) by A. Antonovsky. Polish adaptation. Przegl Psychol 1993;36(4):491-502 [in Polish].

This work is available in Open Access model and licensed under a Creative Commons Attribution-NonCommercial 3.0 Poland License - http://creativecommons.org/ licenses/by-nc/3.0/pl/deed.en. 